\title{
Efeito do Treinamento Físico de Membros Superiores Aeróbio de Curta Duração no Deficiente Físico com Hipertensão Leve
}

\author{
Sandra Haddad, Paulo Roberto Santos Silva, A ntonio Carlos Pereira Barretto, Ivan Ferraretto
}

São Paulo, SP

\begin{abstract}
Objetivo - Verificar as alterações provocadas pelo treinamento físico (TF), com membros superiores (MMSS), em condição aeróbia de curta duração, sobre variáveis dos sistemas cardiovascular e metabólico.

Métodos - Foram estudados 11 deficientes físicos (DF) paraplégicos, com média de idade de 59 anos, sendo 7 homens e 4 mulheres, com lesão de T9 a T11 e grau leve de hipertensão arterial sistêmica (HAS). Os DF foram avaliados antes e após 12 semanas de um programa de TF supervisionado de MMSS, por meio de teste ergométrico (TE) em cicloergômetro mecânico adaptado para MMSS, utilizando-se protocolo intermitente com incremento de carga de $125 \mathrm{kgm} / \mathrm{min}$ (20w) para mulheres e $140 \mathrm{kgm} / \mathrm{min}$ (25w) para homens a cada 3 min., em velocidade que variou de 83 a 95rpm. O consumo de oxigênio $\left(\mathrm{VO}_{2}\right)$ foi calculado de acordo com a equação para MMSS do American College of Sports Medicine. A intensidade do exercício durante o programa de TF foi estabelecida pela reserva de freqüencia cardiaca $(R F C)$ de Karvonen, com variação de $65 \%$ a $85 \%$ e escala de percepção subjetiva ao esforço de Borg obtida pelo TE.

Resultados - A capacidade aeróbia máxima estimada aumentou $22 \%$ (930 349 vs $1138 \pm 290 \mathrm{~mL} / \mathrm{min}$; $p=0,003)$; a pressão arterial sistólica $(P A S)$ e diastólica (PAD) em repouso e no exercício submáximo diminuíram em $4 \%, 15 \%$ e $5 \%, 5 \%$, respectivamente.

Conclusão - O TF aeróbio de intensidade leve a moderada com MMSS, além de melhorar a aptidão funcional exerce também papel importante como modelo terapêutico não medicamentoso na resposta hipertensiva observada em DF paraplégicos.
\end{abstract}

Palavras-chave: hipertensão arterial sistêmica, exercício físico, deficiente físico, paraplegia

\section{The Effect of Short Term Aerobic Physical Training Using Upper Limbs in Paraplegic Persons with Mild to Moderate Hypertension}

Purpose - To study the modifications induced by physical training (PT) using upper limbs in aerobic condition of short duration on cardiovascular and metabolic variables.

Methods - Eleven paraplegic persons, with a mean age of 59 years, 7 men and 4 women with lesions at T9 through T11 and mild hypertension (HAS) were studied. The patients were studied before and after 12 weeks of a supervised program of $P T$, through exercise tests in a mechanic ergometer (ST) adapted for the arms. We emploied an intermittent protocol with stress increments of 125 (20w) and $140 \mathrm{kgm} / \mathrm{min}$ (25w) for women and men, respectively every $3 \mathrm{~min}$. The ergometric speed ranged from 83 to $95 \mathrm{rpm}$. The oxygen consumption $\left(\mathrm{VO}_{2}\right)$ was estimated according to American College of Sports Medicine equation for arms. The exercise intensity during the TF program was estimated through the Karvonen cardiac frequency reserve, with a variation of $65 \%$ to $85 \%$ and the Borg stress scale of subjetive perception during the stress test.

Results - The mean maximal aerobic capacity increased in 22\% (930+349 vs $1138 \pm 290 \mathrm{~mL} / \mathrm{min}$; $p=0.003)$; there was a reduction of systolic and diastolic pressures at rest and after submaximal exercise of 4\%,15\% and $5 \%$ and $5 \%$, respectively.

Conclusion - Physical training of mild to moderate intensity using the upper limbs in paraplegic persons, besides inducing functional capacity increment, is an important model of non drug control of the hypertensive response.

Key-words: hypertension, physic exercise, physical deficient, paraplegia

Arq Bras Cardiol, volume 69 (n 3), 169-173, 1997

Associação de Assistência à Criança Defeituosa e Instituto do Coração do Hospital das Clínicas - FMUSP

Correspondência: Sandra Haddad - Incor - Cardiologia Social - Av. Dr. Enéas C. Aguiar, 44 - São Paulo, SP - 05403-000 - São Paulo, SP

Recebido para publicação em 7/197

Aceito em 2/7/97
A hipertensão arterial sistêmica (HAS) e o sedentarismo são considerados fatores de risco para o desenvolvimento de doenças cardiovasculares. A HAS é uma doença em geral silenciosa, constituindo-se em importante determinante de morbidade e mortalidade cardiovascular. 
Em seguimento de 4 anos, no ambulatório da Associação de Assistência à Criança Defeituosa (AACD), pudemos verificar a incidência de HAS em $25 \%$ dos indivíduos paraplégicos.

Estudos anteriores ${ }^{1,2}$ demonstraram que o risco relativo de morte aumenta em até $70 \%$ em portadores de HAS estabelecida e, quando associado a estilo de vida sedentária, este risco aumenta em cerca de $25 \%{ }^{3,4}$.

Os deficientes físicos (DF) paraplégicos, em geral, apresentam maior número de fatores de risco, pois, pela necessidade de modificarem seus hábitos de vida, aumentam usualmente de peso e tornam-se sedentários, o que favorece ainda mais o desenvolvimento de doenças do aparelho cardiovascular.

Programas de atividades físicas têm sido empregados em estudos epidemiológicos sobre o desenvolvimento de HAS, na tentativa de atenuar a resposta hipertensiva ${ }^{5,6}$

Assim, em vários estudos em homens ${ }^{7,8}$ e animais 9,10 têm se empregado o exercício dinâmico de curta duração, por se constituir em modelo interessante de estudo das adaptações cardiovasculares, induzidas de modo agudo e crônico.

Neste trabalho procuramos verificar os efeitos do treinamento físico (TF) aeróbio de curta duração, com membros superiores (MMSS), sobre variáveis dos sistemas cardiovascular e metabólico, em DF paraplégicos com HAS leve.

\section{Métodos}

Foram estudados 11 DF paraplégicos com nível de lesão de T9 a T11 e grau leve de HAS, com média de idade de 59 anos, sendo sete do sexo masculino e quatro do feminino. Todos foram avaliados antes e após 12 semanas de um programa de TF supervisionado com MMSS.

Previamente ao TF, foram submetidos a eletrocardiograma (ECG) de 12 derivações em repouso com eletrocardiógrafo de inscrição a tinta (Dixtal, modelo Cardiopágina DX 2500) e a estudo ecocardiográfico, com ecocardiógrafo Aloka, modelo SSD 870 (com Doppler pulsado contínuo e mapeamento de fluxo a cores), para diagnosticar e excluir do programa pacientes com possíveis alterações que pudessem interferir no estudo.

Antecedendo ao programa de $\mathrm{TF}$, os DF realizaram teste ergométrico (TE) em cicloergômetro mecânico (Monark), adaptado para MMSS. A engrenagem e circunferência do volante desse ergômetro foram dimensionadas de modo que, uma volta completa dos pedais desenvolvesse uma distância teórica comparável a $6 \mathrm{~m}$. O volante era freado mecanicamente por uma correia que se situava em volta do aro. Ambas as extremidades dessa correia eram presas a um tambor giratório ao qual estava fixado um pêndulo. Agindo assim, como uma balança de pêndulo, media-se a diferença de força em cada ponta da correia. A escala de graduação é em kilopounds (kp) (1kpé a força que age sobre uma massa de $1 \mathrm{~kg}$ ) à aceleração normal da gravidade. O poder de frenagem (kp) estipulado pelo ajuste da tensão da correia, multiplicado pela distância pedalada ou ciclada (m), fornece o montante de trabalho em kilopounds/metros (kpm) ou kilograma/metros (kgm). Se a distância for expressa pelo tempo gasto na realização do exercício, então, obtém-se a média do trabalho em $\mathrm{kpm} / \mathrm{min}^{-1}$ ou $\mathrm{kgm} / \mathrm{min}^{-1}$. Os testes foram realizados de acordo com protocolo elaborado pelo Departamento de Cardiologia. OTE intermitente foi iniciado com carga livre ou carga equivalente a aproximadamente $55 \mathrm{kgm} / \mathrm{min}^{-1}(9 \mathrm{w})$. Posteriormente, foi utilizado incremento de carga de $125 \mathrm{kgm} / \mathrm{min}^{-1}(20 \mathrm{w})$ e $140 \mathrm{kgm} / \mathrm{min}^{-1}(25 \mathrm{w})$, para mulheres e homens, respectivamente. Cada estágio teve duração de $3 \mathrm{~min}$, intervalo ativo de $1 \mathrm{~min}$ e velocidade individualizada, variando de 83 a 95rpm..

A freqüência cardíaca (FC) foi monitorizada continuamente em repouso, durante o esforço e na fase de recuperação por monitor acoplado ao ECG com três derivações simultâneas $\left(\mathrm{MC}_{5}, \mathrm{AV}_{\mathrm{F}} \mathrm{e} \mathrm{V}_{2}\right.$ ) registradas a cada minuto.

As pressões arteriais sistólica (PAS) e distólica (PAD) foram medidas por método auscultatório, utilizando-se esfigmomanômetro aneróide (Tycos) em repouso, no final de cada estágio do TE e durante o intervalo ativo. A medida de PA durante o intervalo ativo, foi verificada em um dos braços, enquanto o outro, continuava ciclando. Os critérios de determinação da PAS e PAD foram, o $1^{\circ}$ e o $5^{\circ}$ som de Korotkoff, respectivamente.

Utilizou-se para se calcular o consumo máximo de oxigênio (VO2máx) para MMSS a equação do American College of Sports Medicine, ou seja, VO2máx $=\left(\mathrm{kgm} / \mathrm{min}^{-1} \mathrm{x}\right.$ $\left.3,0 \mathrm{mLO} / \mathrm{kg}^{-1} \mathrm{~min}^{-1}\right)+\left(\text { peso }[\mathrm{kg}] \times 3,5 \mathrm{mLO}_{2} / \mathrm{kg}^{-1} \mathrm{~min}^{-1}\right)^{11}$.

O regime de TF foi iniciado logo após o TE de MMSS, sendo a intensidade inicial de exercício no cicloergômetro com MMSS de $65 \%$ da reserva de FC preconizada por Karvonen e ajustada ao longo do TF ao máximo de $85 \%$ obtida pelo $\mathrm{TE}^{12}$. Foi utilizada para ajustar a progressão do $\mathrm{TF}$, a escala de percepção subjetiva ao esforço de Borg até o período final do programa ${ }^{13,14}$.

Cada sessão consistiu de aquecimento ( $5 \mathrm{~min})$, exercício no cicloergômetro (30min) e volta à calma (15min). Foram realizadas três sessões semanais até o fim do programa que durou 12 semanas.

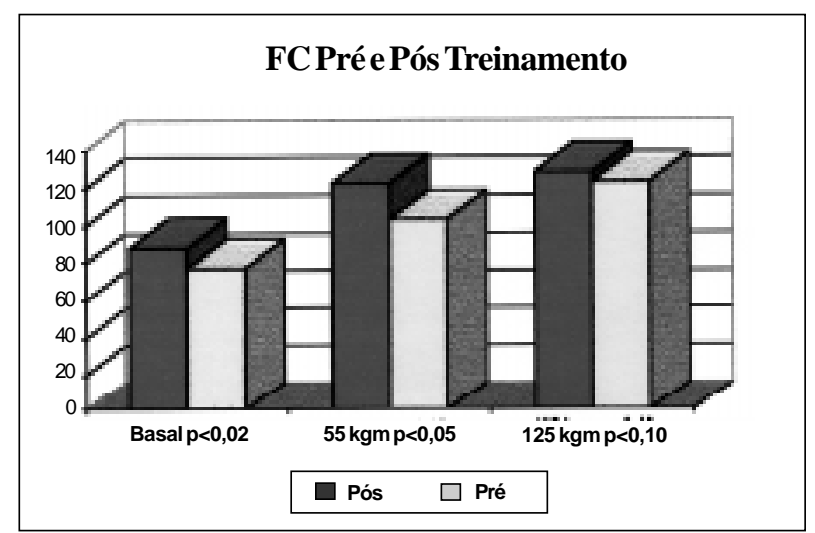

Fig. 1 - Resposta da freqüência cardíaca pré e pós treinamento físico em repouso e no exercício submáximo. 


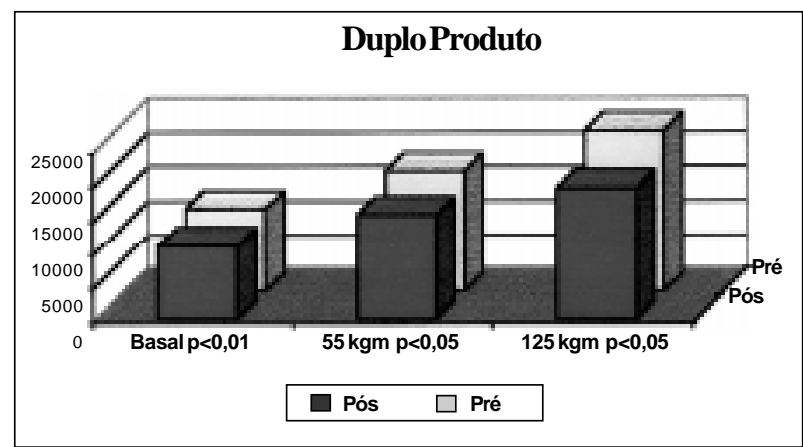

Fig. 2 - Duplo produto pré e pós treinamento físico em repouso e no exercício submáximo.

\begin{tabular}{|c|c|c|}
\hline \multicolumn{3}{|c|}{$\begin{array}{l}\text { Tabela I - Resultados de freqüência cardíaca (FC), pressão arterial } \\
\text { sistólica (PAS), pressão arterial diastólica (PAD) e duplo produto } \\
\text { (DP) em repouso antes e após treinamento físico (TF) de membros } \\
\text { superiores (MMSS) }\end{array}$} \\
\hline Variável & Pré - TF & Pós - TF \\
\hline $\mathrm{FC}(\mathrm{bpm} / \mathrm{min})$ & $88 \pm 3$ & $76 \pm 12 *$ \\
\hline PAS (mmHg) & $140 \pm 20$ & $134 \pm 16 * *$ \\
\hline PAD (mmHg) & $100 \pm 13$ & $85 \pm 09 * *$ \\
\hline $\mathrm{DP}(\mathrm{PAS} \times \mathrm{FC} / 100)$ & $120 \pm 29$ & $108 \pm 30 * *$ \\
\hline
\end{tabular}

\begin{tabular}{|c|c|c|}
\hline \multicolumn{3}{|c|}{$\begin{array}{l}\text { Tabela II - Resultados de freqüência cardíaca (FC), pressão arterial } \\
\text { sistólica (PAS), pressão arterial diastólica (PAD) e duplo produto } \\
\text { (DP) no exercício submáximo }\left(55 \mathrm{kgm} / \mathrm{min}^{-1}\right) \text { antes e após treinamento } \\
\text { físico (TF) de membros superiores (MMSS) }\end{array}$} \\
\hline Variável & Pré-TF & Pós-TF \\
\hline $\mathrm{FC}(\mathrm{bpm} / \mathrm{min})$ & $112 \pm 29$ & $102 \pm 18^{*}$ \\
\hline PAS (mmHg) & $163 \pm 19$ & $155 \pm 5 * * *$ \\
\hline $\mathrm{PAD}(\mathrm{mmHg})$ & $103 \pm 9$ & $98 \pm 8^{*}$ \\
\hline $\mathrm{DP}(\mathrm{PAS} \times \mathrm{FC} / 100)$ & $182 \pm 45$ & $158 \pm 29 * *$ \\
\hline
\end{tabular}

\begin{tabular}{|c|c|c|}
\hline \multicolumn{3}{|c|}{$\begin{array}{c}\text { Tabela III - Resultados do tempo de tolerância (TT), consumo de } \\
\text { oxigênio }\left(\mathrm{VO}_{2}\right) \text {, carga de trabalho (CT), freqüência cardíaca (FC) e } \\
\text { pulso de oxigênio }\left(\mathrm{PO}_{2}\right) \text { no exercício máximo antes e após } 12 \text { semanas } \\
\text { de treinamento físico (TF) de membros superiores (MMSS) }\end{array}$} \\
\hline Variável & Pré-TF & Pós-TF \\
\hline $\mathrm{TT}(\mathrm{s})$ & $532 \pm 187$ & $644 \pm 152 *$ \\
\hline $\mathrm{CT}(\mathrm{kgm} / \mathrm{min})$ & $224 \pm 118$ & $298 \pm 96 * * *$ \\
\hline $\mathrm{VO}_{2}(\mathrm{~mL} / \mathrm{min})$ & $930 \pm 349$ & $1138 \pm 290 * *$ \\
\hline $\mathrm{PO}_{2}\left(\mathrm{VO}_{2} / \mathrm{FC}\right)$ & $6,98 \pm 2,95$ & $8,36 \pm 2,55^{* *}$ \\
\hline $\mathrm{FC}(\mathrm{bpm})$ & $139 \pm 28$ & $141 \pm 27$ \\
\hline \multicolumn{3}{|c|}{ Teste t pareado $* \mathrm{p}<0,05 ; * * \mathrm{p}<0,03 ; * * * \mathrm{p}<0,002$} \\
\hline
\end{tabular}

Treinamento físico de membros superiores no deficiente físico

O estudo estatístico constituiu-se no cálculo da média e desvio padrão das variáveis numéricas. Para verificar se as variáveis analisadas diferiram nos períodos pré e pós TF, utilizou-se o teste de Student para dados pareados. Para fins de análise, foi considerado o nível de significância $\mathrm{p}<0,05^{15}$.

\section{Resultados}

Os DF terminaram o período de 12 semanas de TF com aderência de $90 \%$. Em repouso, verificaram-se diminuição da FC em $14 \%$ (88 vs 76bpm), (fig. 1), do duplo produto em $10 \%$ (120 vs 108 PAS x FC/100), (fig. 2) enquanto as PAS e PAD diminuíram $6 \mathrm{mmHg}(4 \%)$ e $15 \mathrm{mmHg}(15 \%)$, respectivamente, após TF (tab. I). Resultados semelhantes foram observados no exercício submáximo $\left(55 \mathrm{kgm} \cdot \mathrm{min}^{-1}\right)$. A FC após o TF nessa intensidade foi reduzida em $9 \%$ (112 vs $102 \mathrm{bpm})$, sendo de $8 \mathrm{mmHg}(5 \%)$ a diminuição das PAS e PAD e de $13 \%$ no DP (tab. II). No exercício máximo, o tempo de tolerância aumentou $21 \%$ (532 vs 644s), a carga máxima de trabalho $33 \%$ (224 vs 298kgm/min); a capacidade aeróbia máxima aumentou $22 \%$ (930vs $1138 \mathrm{mLO}_{2} / \mathrm{min}$ ) e o pulso de oxigênio $19 \%$ (6,09 vs 9,36mLO_/FC). A FC máxima atingida não foi modificada (tab. III).

\section{Discussão}

Nosso objetivo foi estudar o efeito do TF com MMSS, como modelo importante de atenuação e/ou controle da HAS de grau leve, em indivíduos DF com paraplegia.

Tem sido comum na rotina clínica, a utilização de terapêutica farmacológica no manuseio de indivíduos com HAS, entretanto, os resultados de vários ensaios randomizados, que demonstraram efeitos indesejáveis no tratamento farmacológico em portadores de HAS, induziram a uma mudança de atitude da comunidade médica no combate a HAS.

Vários são os estudos que demonstraram evidências favoráveis do efeito anti-hipertensivo do TF com características aeróbias, entretanto, ainda existem controvérsias sobre os meios e controles utilizados nos programas de atividade física para hipertensão, a metodologia mais adequada para a avaliação das intensidades de exercício e os possíveis mecanismos responsáveis pela atenuação desta síndrome cardiovascular ${ }^{16-24}$. Embora o efeito crônico do TF na PA de indivíduos normotensos seja controversa, a maioria dos estudos tem demonstrado que o exercício aeróbio diminui significativamente a pressão arterial (PA) de repouso ${ }^{21,22,24,25}$.

Apesar dos mecanismos que determinam a diminuição da PA após uma única sessão de exercício ainda não estarem totalmente esclarecidos, parece que a atenuação da atividade nervosa simpática periférica pode explicar parcialmente essa resposta no exercício agudo ${ }^{26}$.

Floras e col ${ }^{26}$ verificaram por técnica de microneurografia, que a diminuição da atividade simpática do nervo peronero em hipertensos limítrofes, observada 60min após uma sessão de exercício físico, coincidia com a diminuição 
da PA. A ativação do sistema de opióides peptídicos tem sido sugerida como um dos prováveis mecanismos responsáveis por essa atenuação da atividade nervosa simpática periférica $^{27}$.

Os mecanismos pelos quais o exercício crônico, a longo prazo, atenuam a hipertensão, também não estão esclarecidos, mas parece que, em parte, eles podem ser explicados por alguns comportamentos: 1) após um programa de $\mathrm{TF}$, a redução da atividade nervosa simpática periférica contribui para a atenuação da PA, visto que, observa-se que os níveis de norepinefrina circulante ${ }^{22,25,28-30}$ estão diminuídos; 2) o aumento da excreção urinária de sódio e, conseqüentemente, a diminuição da atividade da renina plasmática ${ }^{21,25}$ são respostas que também têm sido utilizadas para explicar a diminuição da PA após TF.

O nosso estudo não apresenta grupo controle, todavia, o grupo comparado antes e após 12 semanas de TF com MMSS, apresentou resultados significativos de melhoria de variáveis cardiovasculares, com conseqüente aumento da capacidade funcional dos paraplégicos.

A bradicardia observada no homem após TF, em condições de repouso, é um achado freqüente, ocorrendo também na presente investigação. Isto evidencia que o TF utilizado constituiu-se de exercício de intensidade predominantemente moderada e de longa duração, pois são exercícios desta intensidade que induzem a diminuição da FC no repouso $^{3,18}$.

No exercício submáximo, com carga de $55 \mathrm{kgm} / \mathrm{min}^{-1}$, a resposta de FC, também foi significativamente menor após $\mathrm{TF}(\mathrm{p}<0,05)$ o que demonstra, provavelmente, aumento da estimulação do sistema nervoso autônomo parassimpático pelo TF aeróbio, com consequiente efeito sobre o cronotropismo cardíaco ${ }^{4,18}$.

Ainda hoje, não existe consenso, sobre os efeitos redutores do TF na PA. Entretanto, alguns estudos têm demonstrado efeito hipotensor provocado pelo exercício regular, independente da perda de peso, como demonstraram Arrol e Beaglehole ${ }^{17}$, que encontraram para PAS e PAD decréscimos de 30 a $20 \mathrm{mmHg}$ em repouso e 25 a $12 \mathrm{mmHg} \mathrm{du-}$ rante o esforço.

Essa queda também foi observada por Seals e Hagberg ${ }^{19}$, que verificaram em 12 estudos publicados entre 1976 e 1983, redução média da PAS e PAD em repouso de 9 e $7 \mathrm{mmHg}$, respectivamente.

Em nosso estudo, obtivemos resultados semelhantes, com diminuição de $6 \mathrm{mmHg}$ para a PAS e $15 \mathrm{mmHg}$ para PAD, em medidas realizadas antes e após exercício.

Sannersted e $\mathrm{col}^{5}$, ao estudarem indivíduos com HAS, não encontraram diferenças no débito cardíaco e na resistência vascular sistêmica, entretanto, mesmo assim, observaram queda da PA em repouso e durante exercício, no valor de $5 \mathrm{mmHg}$.

Clausen ${ }^{6}$ postula que a diminuição da PA com o TF no repouso e durante exercício poderia ser explicada por queda de débito cardíaco ou redução na resistência periférica total ou ainda de ambos, acreditando que com a atividade física ocorra redução da estimulação simpática após TF, induzindo benefícios aos indivíduos com HAS.

Recentemente, Shenberg e col ${ }^{20}$ estudando em paraplégicos submetidos a TF, o efeito do exercício crônico na dinâmica dos vasos de condução, concluíram que a atividade física crônica de MMSS provocou melhora na capacidade vasodilatadora e aumento da condutância dos vasos em repouso e durante exercício, quando comparado a um grupo controle. Esses resultados foram obtidos através do teste de hiperemia reativa, permitindo aos autores ${ }^{20}$ confirmar uma das hipóteses de Clausen ${ }^{6}$, que acredita na diminuição da resistência periférica total, como possível determinante do efeito da TF, na redução da PA.

Além dos efeitos verificados sobre a PA dos DF, nosso estudo mostrou que o TF aeróbio com MMSS foi um estímulo eficiente para aumentar o tempo de tolerância ao exercício e carga máxima de trabalho(CT), além do incremento no pulso de oxigênio $\left(\mathrm{PO}_{2}\right)$ e melhorar o consumo máximo de oxigênio(VO2máx) (tab. III).

Neste estudo, não tivemos a intenção de estudar todos os possíveis mecanismos envolvidos na redução da PA, especialmente considerando-se as controvérsias discutidas na literatura. Entretanto, o TF nesses indivíduos é de grande importância, pois pode possibilitar a diminuição da dose de drogas anti-hipertensivas a que a maioria dos DF hipertensos está sujeita ou, até mesmo, a supressão do uso delas durante o tratamento de HAS.

Portanto, é fato que os resultados obtidos neste trabalho demonstraram efeitos favoráveis, que nos permitem acreditar e indicar que o exercício aeróbio controlado, além de melhorar a aptidão funcional, exerce também, importante papel como modelo terapêutico não medicamentoso na possível atenuação da resposta hipertensiva de grau leve, em DF paraplégicos submetidos a TF com MMSS.

\section{Referências}

1. Paffenberger JSRS, Hyate RT, Wing AL et al - The association of changes in physical activity level and other lifestyle characterystics with mortality among men. NEng J Med 1993; 328: 536-45.

2. Sandvik L, Erikssen J, Thanlw Eet al - Physical fitness as a predictor of mortality among healthy, middle-aged Norwegian men. N Engl J Med 1993; 328:533-7.

3. Gallo Jr L, Maciel BC, Marin-Neto JA et al - Sympathetic and parasympathetic changes in heart rate control during dynamic exercise induced by endurance training in man. Braz J Med Res 1989; 22: 631-43.
4. Hartley LH, Grimby A, Kilbom NJ et al - Physical training in sedentary midlle aged and old men. III. Cardiac output and gas exchange at submaximal an maximal exercise. Scand J Clin Lab Invest 1969; 24: 334-5.

5. Sannersted R, Wasir H, Henning Retal - Systemic hemodynamics mild arterial hypertension before and after physical training. Clin Sci Mol Med 1973; 45: 145-9.

6. Clausen GP-Circulatory adjustment to dynamic exercise and effects of physical training in normal subjects and in patients with coronary artery disease. Prog Cardiovasc Dis 1976; 18: 459-95. 
7. Mann GV, Garrett HL, Farhi A et al - Exercise to prevent coronary heart disease. An experimental study of the effects of training on risk factory for coronary disease in men. Am J Med 1969; 46: 12-27.

8. Hagberg JM, Montain SJ, Martin WHet al - Effects of exercise training in 60 to 69year old persons with essencial hypertension. Am J Cardiol 1989: 64: 348-53.

9. Ghaemmaghami F, Sassolas A, Gauquelin G et al - Swim training in genetically hypertensive rats of the lyon strain: effects on plasma lipids and lipoproteins. J Hypertension 1986; 4: 319-24.

10. Marcus KD, Tipton CM - Exercise training and its effects with renal hypertensive rats. J Appl Physiol 1985; 59: 1410-15.

11. Guidelines for Exercise Testing and Prescription - American College of Sports Medicine. $3^{\text {rd }}$ ed. Philadelphia: Lea \& Fabiger, 1986; 168.

12. Karvonen MJ, Kentala E, Mustala O - The effects of training on heart rate. A longitudinal study. Ann Med Exper Fenn 1957; 35: 307-15.

13. Dunbar CC, Robertson RJ, Baun Retal - The validity of regulating exercise intensity by ratings of perceived exertion. Med Sci Sports Exerc 1992; 24: 94-9.

14. Dishman RK, Patton RW, Smith J et al - Using perceived exertion to prescribe and monitor exercise training heart rate. Int J Sports Med1987; 8: 208-13.

15. Roger E, Kirk - Experimental Design Procedures for the Behavioral Sciences Statistical Tables for Biological, Agricultural and Medical Research, $2^{\text {nd }}$ ed. $1983 ; 813$.

16. Harsfield GA, DupaulLM, Alfort BS-Aerobic fitness and the diurnal rhythm of flod pressure in adolescents. Hypertension 1990; 15: 810-14

17. Arrol B, Beaglehole R - Does physical activity lower blood pressure?: A critical review of the clinical trials. J Clin Epidemiol 1992; 45: 439-47.

18. Katona PG, Mc Lean M, Dighton DHet al-Sympathetic and parasympathetic cardiac control in athletes and nonathletes at rest. J Appl Physiol 1982; 52: 1652-7.
19. Seals DR, Hagberg JM - The effects exercise training on human hypertensive: A review. Med Sci Sports Exerc 1984; 16: 207-15.

20. Shenberger JS, Leaman GJ, Neumyer MM et al - Physiologic and structural in dices of vascular function in paraplegics. Med Sci Sports Exerc 1990; 22: 96 101.

21. Fagart R-Habitual physical activity, and blood pressure in normal and hypertension. Int J Sports Med 1985; 6: 57-67.

22. Bjorntorp $\mathrm{P}$ - Effects of physical training on blood pressure in hypertension. Eur Heart J 1987; 8(suppl. 3): 71-6.

23. Cade R, Mars D, Wagemaker Het al -Effect of aerobic exercise training on patient with systemic arterial hypertension. Am J Med 1984; 77: 785-90.

24. Franz IW - Blood pressure response to exercise in normotensive and hypertension. Int J Sports Med 1989; 10: 596.

25. Kiyonaga A, Arakawa K, Tanaka H, Shindo M - Blood pressure and hormona responses to aerobic exercise. Hypertension 1985; 7: 125-31.

26. Floras JS, Sinkey CA, Ayleard PE et al - Post exercise hypertension and sympathoinhibition in borderline hypertensive men. Hypertension $1989 ; 14$ 28-35.

27. Thoren P, Floras JS, Hofmann P et al - Endorphins and exercise: physiological mechanisms and clinical implications. Med Sci Sports Exerc 1990; 22: 417-28.

28. Hartley LH, Mason JW, Hogan RP et al - Multiple hormonal responses to graded exercise in relation to physical training. J Appl Physiol 1972; 33: 602-6.

29. LeBlanc J, Boulay M, Dulac S et al - Metabolic and cardiovascular responses to norepinephrine in trained and nontrained humans subjects. J Appl Physiol 1977; 42: 166-73.

30. Peronnet F, Cleroux J, Perrault H et al - Plasma norepinephrine response exercise before and after training in humans. J Appl Physiol 1981; 51: 812-15. 